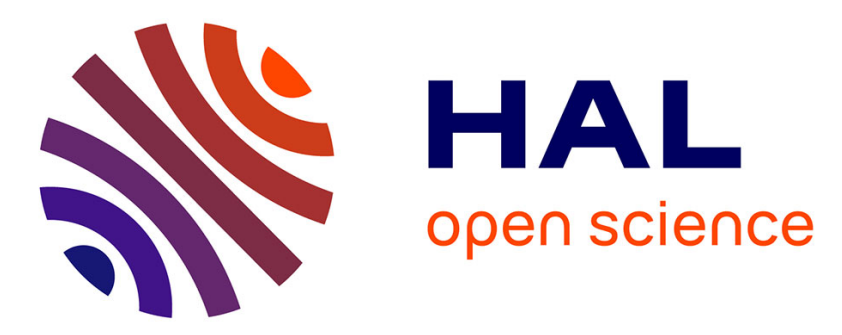

\title{
Light Sources and Cameras for Standard in Vitro Membrane Potential and High-Speed Ion Imaging
}

\author{
R. Davies, J. Graham, M. Canepari
}

\section{To cite this version:}

R. Davies, J. Graham, M. Canepari. Light Sources and Cameras for Standard in Vitro Membrane Potential and High-Speed Ion Imaging. Journal of Computer-Assisted Microscopy, 2013, 251 (1), pp.8. 10.1111/jmi.12047 . hal-01137829

\section{HAL Id: hal-01137829 \\ https://hal.science/hal-01137829}

Submitted on 31 Mar 2015

HAL is a multi-disciplinary open access archive for the deposit and dissemination of scientific research documents, whether they are published or not. The documents may come from teaching and research institutions in France or abroad, or from public or private research centers.
L'archive ouverte pluridisciplinaire HAL, est destinée au dépôt et à la diffusion de documents scientifiques de niveau recherche, publiés ou non, émanant des établissements d'enseignement et de recherche français ou étrangers, des laboratoires publics ou privés. 


\title{
Light Sources and Cameras for Standard in Vitro Membrane Potential and High-Speed Ion Imaging
}

\author{
Rosanna Davies ${ }^{1}$, Jeremy Graham ${ }^{1}$ and Marco Canepari ${ }^{2,3,4}$
}

${ }^{1}$ CAIRN Research Ltd., Faversham, UK

${ }^{2}$ Inserm U836, Team 3, Grenoble Cedex 09, France

${ }^{3}$ Université Joseph Fourier, Grenoble Institut des Neurosciences et Laboratoire Interdisciplinare de Physique (CNRS UMR 5588), France

${ }^{4}$ Laboratories of Excellence, Ion Channel Science and Therapeutics

Corresponding author: Marco Canepari, INSERM U836 - Grenoble Institute of Neuroscience, Bâtiment Edmond Safra, Chemin Fortune Ferrini, Site santé de la Tronche - BP 170, 38042 Grenoble cedex 9, France. Email: marco.canepari@ujf-grenoble.fr

Keywords: Voltage imaging; Calcium imaging, CCD cameras, CMOS cameras, laser, LED 


\begin{abstract}
Membrane potential and fast ion imaging are now standard optical techniques routinely used to record dynamic physiological signals in several preparations in vitro. Whereas detailed resolution of optical signals can be improved by confocal or two-photon microscopy, high spatial and temporal resolution can be obtained using conventional microscopy and affordable light sources and cameras. Thus, standard wide-field imaging methods are still the most common in research laboratories and can often produce measurements with a signal-to-noise ratio that is superior to other optical approaches. This paper seeks to review the most important instrumentation used in these experiments, with particular reference to recent technological advances. We analyse in detail the optical constraints dictating the type of signals that are obtained with voltage and ion imaging and we discuss how to use this information to choose the optimal apparatus. Then, we discuss the available light sources with specific attention to LEDs and solid state lasers. We then address the current state-of-the-art of available CCD, EMCCD and CMOS cameras and we analyse the characteristics that need to be taken into account for the choice of optimal detector. Finally, we conclude by discussing prospective future developments that are likely to further improve the quality of the signals expanding the capability of the techniques and opening the gate to novel applications.
\end{abstract}




\section{Introduction}

In physiological research, optical recordings of ion concentration and membrane potential changes $\left(\Delta \mathrm{V}_{\mathrm{m}}\right)$ are now routinely taken in many laboratories (see Helmchen \& Konnerth Eds, 2011). Whereas confocal and two-photon (2P) microscopy are used to localise signals in three dimensions, standard imaging measurements using wide-field illumination and camera detection are most commonly used and remain preferable in many situations for several reasons. First, they allow fast measurements from many sites with a spatial resolution, in principle, limited only by the size and number of pixels of the camera. Second, both charge coupled device (CCD) and complementary metal oxide semiconductor (CMOS) cameras have high performance in relation to these types of measurement; in particular they are efficient at capturing photons at all visible wavelengths, they have low intrinsic (read-out) noise and they are capable of reaching full-frame acquisition rates in the order of kHz. Third, the accuracy of widefield measurements is often superior compared with confocal or $2 \mathrm{P}$ imaging because of the higher signal-to-noise ratio $(\mathrm{S} / \mathrm{N})$ achievable. Finally, the instrumentation is generally cheaper, easier to install and more user-friendly.

In vitro or ex-vivo optical recordings, mainly used in brain (Homma et al., 2009) and heart research (Efimov et al., 2005), are performed in culture preparations, in tissue slices (for example in acute brain slices, Grinvald et al., 1982; Augustine 1994), or in intact organs (for example in the heart, Walton et al., 2010). In general optical measurements can be divided into two categories: those performed on single cells typically individually stained with dyes dissolved in the intracellular solution and loaded via a sharp or patch-clamp electrode (see for example Canepari et al., 2008); and those performed on multiple cells either stained extracellularly with AM-forms of ion indicators and voltage sensitive dyes or genetically encoding $\mathrm{Ca}^{2+}$ indicators (Miyawaki et al., 1997) or more recent voltagesensitive proteins (Mutoh et al., 2011; Jin et al., 2012). The feasibility of calibrating signals, as well as recording the amplitude of an optical signal corresponding to a given physiological signal, depends on the type of experiment. In order to optimise the instrumentation it is important to understand the characteristics of the biological preparation. For example, photons emitted by single cells may be substantially fewer than those emitted by a stained tissue illuminated at the same intensity and wavelength. In contrast, the fractional change of fluorescence $(\Delta F / F)$ corresponding to a given physiological signal can be significantly larger in the case of a single emitting cell.

The performance of commercially available illumination and detection systems has improved significantly in the last decade. We will provide an overview of the currently available instruments, analysing in detail advantages and disadvantages of different devices in relation to the most common applications. In analysing the light source, we will start with a historical overview to describe the 
technological developments that led to the transition from lamps, which were typically used until 10 years ago, to systems based on light emitting diodes (LEDs) or solid-state lasers. In the same way, in the following section, we will describe the progression from cameras based on photodiode arrays and "slow" CCD cameras, to fast CCD cameras, electron multiplying CCD (EMCCD) cameras and CMOS cameras. Finally, we will discuss the future of the instrumentation, highlighting technologies which will become available to researchers in the next few years.

\section{Needs and constraints in voltage and ion imaging applications}

In designing the optimal apparatus for a particular voltage or ion fluorescence measurement, two important features of the optical signal must be primarily taken into account: the amplitude of the signal, in terms of fractional change of fluorescence $(\Delta \mathrm{F} / \mathrm{F})$, and the kinetics of any physiological changes. It is obvious that, ideally, one wants to maximize the size of the signal; in practise the optimal measurement is the one that maximizes the $\mathrm{S} / \mathrm{N}$. As a practical example, it is useful to see the difference of performing $\mathrm{Ca}^{2+}$ imaging using different indicators. Experiments using Fluo indicators are characterised by poor background fluorescence but large dynamic range permitting high $\Delta \mathrm{F} / \mathrm{F}$ signals (Harkins et al., 1993); in contrast, Calcium-Green or Oregon Green have larger background fluorescence but smaller dynamic range (Thomas et al., 2000). The size of $\Delta \mathrm{F} / \mathrm{F}$ signals will also depend on the absorption and emission spectra of the indicator and the extent to which wavelengths can be optimised. This is particularly the case for ratiometric ion indicators and for several voltage sensitive dyes. Fig. 1 illustrates the case of the styryl voltage sensitive dye JPW1114 excited at $532 \mathrm{~nm}$ by a laser and at the 500-550 $\mathrm{nm}$ wavelength band by the filtered light of a xenon lamp (Canepari et al., 2010). For this type of dye, a membrane potential depolarisation produces a red-shift of the absorption spectrum. Thus, the $\Delta \mathrm{F} / \mathrm{F}$ signal is $\sim 2.5$ larger when fluorescence is excited at a narrow band at the edge of the dye absorption spectrum $(532 \mathrm{~nm})$ than with a large band closer to the dye absorption peak.

It is useful here to consider that a light measurement is always associated with an intrinsic photon noise and at constant conditions the number of measured photons fluctuates from a mean value $\langle\mathrm{M}\rangle$; the standard deviation (SD) of these fluctuations is given by the square root of the mean number of measured photons. Because in an imaging experiment one wishes to measure a percentage change in the number of photons, i.e. a $\Delta \mathrm{F} / \mathrm{F}$ signal, it is necessary that this change is larger than the fluctuations of the photon noise. If the threshold for a signal to be detectable over the noise is set to 3 times the SD of the photon noise (i.e. $\mathrm{S} / \mathrm{N} \geq 3$ ), the smallest detectable $\Delta \mathrm{F} / \mathrm{F}$ will be $30 \%$ if $\langle\mathrm{M}\rangle=100$ photons, it will be $3 \%$ if $\langle\mathrm{M}\rangle=10^{4}$ photons and it will be $0.3 \%$ if $\langle\mathrm{M}\rangle=10^{6}$ photons. Thus, larger $\Delta \mathrm{F} / \mathrm{F}$ signals can be measured with less background fluorescence. More photons can be obtained by increasing the 
power of illumination, but also by summing photons from rectangular arrays of pixels, a process called binning, or from adding consecutive frames, in this way decreasing either the spatial or the temporal resolution of the measurement. The golden rule of "more photons are necessary to measure a smaller $\Delta \mathrm{F} / \mathrm{F}$ signal" is therefore associated with the effective spatial and temporal resolution of the detectable signal but is also crucial when choosing the optimal camera. Thus, a first parameter to take into account is the well-depth (or well-capacitance), i.e. the maximum number of electrons $\left(\mathrm{e}^{-}\right)$per pixel that can be excited by photon absorption. This will set the smallest $\Delta \mathrm{F} / \mathrm{F}$ signal that can be measured by a single pixel in a single trial. For example if the well-depth is $=10^{4}$, it is evident that signals $<3 \%$ can be measured only by averaging fluorescence over several pixels or by averaging several trials to obtain a total background fluorescence corresponding to a number of photons larger than the welldepth. For detectors that can operate at higher speeds than the required measurements the temporal binning (or frame averaging) can also be used to increase the effective well-depth. As different detectors can and do have a wide range of pixels sizes it is important to consider the well-depth per unit area in addition to the well-depth per pixel when assessing the achievable spatial resolution. The second parameter to take into account is the read-out noise, which is the additional noise of the camera associated with the capture and digitisation of each frame. The goal is always to have a photon noise significantly higher than the read-out noise, i.e. to be limited by the physics of the measurement and not by the apparatus. Thus, if the read-out noise is $20 \mathrm{e}^{-}$, the camera is adequate to measure a $\Delta \mathrm{F} / \mathrm{F}$ signal of $1 \%$ with a background fluorescence of $10^{5}$ photons (i.e. a signal of $10^{3}$ photons), but it will not be adequate to measure a $\Delta \mathrm{F} / \mathrm{F}$ signal of $20 \%$ with a background fluorescence of 400 photons. An example of photon noise limited recording of a $\mathrm{Ca}^{2+} \Delta \mathrm{F} / \mathrm{F}$ signal is reported in Fig.2. In this case, the recording is taken at 2,000 frames/s and at this speed the read-out noise is $12 \mathrm{e}^{-}$. Thus, the light in the sample pixel reported in Fig. 2 corresponding to 2,500 photons will have a photon noise $=50$ photons that will dominate the read-out noise of the camera. Finally, it is also necessary to take into account the number of bits that each pixel is digitised to, typically $\geq 12$ to produce $>4000$ discrete levels of fluorescence. In the case of the recording of Fig.2, the signal was digitised to 14 bits.

The optimal measurement is also the one in which the detector is sampling the fluorescence change fast enough to measure its kinetics. As with electrical recordings, the sampling frequency must be at least twice the maximal frequency component of the signal to avoid distortion. Thus, whereas the recording of an action potential in the dendrite can be often achieved at $2-5 \mathrm{kHz}$ (Canepari et al., 2007), the measurement of axonal action potentials requires higher acquisition frequencies (Foust et $a l ., 2010)$. In addition to matching the temporal resolution to the kinetics it is important to ensure that the sensor size, pixel size, and number of pixels are sufficient to resolve the required field of view in enough detail to answer the physiological question being addressed. 


\section{Light sources}

\section{Historical overview of light sources for voltage and ion imaging}

Ideal light sources for membrane potential and ion imaging must provide high intensities of illumination at particular wavelengths and must be stable, with fluctuations of less than $0.1 \%$. Until recently, the most commonly used light sources were lamps. Tungsten and halogen lamps, an economic solution to obtain white stable light that could band-filtered in the visible range, were used for pioneering studies of membrane potential and $\mathrm{Ca}^{2+}$ imaging (see for example Ross et al., 1974; Brown et al., 1975). These were progressively replaced by stabilised xenon, mercury and metal halide arc lamps offering somewhat reduced stability, but much higher point intensities of illumination (Thomson \& Hageage, 1975). Xenon lamps produce a uniform spectrum permitting selection of any wavelength in the near-infrared, visible and near-ultraviolet range. For this reason, they are also used as light sources in fast monochromators such as the Polychrome (TILL Photonics GmbH, Gräfelfing, Germany) and the Optoscan (Cairn Research Ltd, Faversham, UK). In contrast to xenon lamps, the spectra of mercury and metal halide lamps are characterised by six major peaks in the ultraviolet visible range. Several of these peaks are potentially useful for ion and membrane measurements, but these lamps are generally less stable than xenon lamps and rarely suitable for physiological studies where signal changes are small. Stable Xenon arc lamps from 100 to 250 Watts are however manufactured by several companies including microscope manufacturers; although these should be tested carefully as many do have residual mains interference or other instability due to arc wander. Despite their high power, the monochromatic (or nearly-monochromatic) light reaching the backaperture of the objective can often be limited in power to a few milliwatts. In addition, arc lamps stabilise in several minutes and they need to be constantly on to be operational; illumination for short time intervals is therefore only achieved using mechanical shutters such as those commercialised by Vincent Associates (Rochester, NY). The need for more powerful and more practical light sources has led researchers to seek alternatives as described below.

\section{LEDs}

In the last decade, stable LED illuminators permitting usable power of tens of milliwatts at many different wavelengths have become an attractive solution for voltage and ion imaging (Entcheva et al., 2004). LED systems are now commercialised by microscope manufacturers as well as by specialist companies such as Cairn Research, Thorlabs (Newton, NJ), CoolLED (UK) and Rapp OptoElectronic (Hamburg, Germany). LED light can be introduced episcopically into all commercial research 
microscopes and the devices thermally stabilised to avoid intensity fluctuations and to protect the diode from heat damage. In addition to the higher practical illumination power achievable, LED systems are more economical than lamps and they have several technical advantages. A first advantage is that LEDs reach their full intensity in microseconds and they can therefore be triggered by a digital TTL pulse. Thus, they don't require shutters and can be modulated to give extremely short exposure times, Although they do usually require optical filtering, to remove residual bleed-through into the emission band, they do not produce significant heat and therefore do not require infra-red filtering. A second advantage is the wide range of colours that are currently available, from ultraviolet to infra-red, having spectral bandwidths ranging between $20 \mathrm{~nm}$ and $200 \mathrm{~nm}$. Because of the low cost and compact size, several LEDs can be cascaded using dichroic beamsplitters permitting simultaneous illumination at different wavelengths. It should be noted that LED intensity at the specimen is largely dictated by the epi-condenser optics and that direct illumination into the objective lens is usually preferable to using the standard microscope epi-condenser which will be optimised for arc lamps rather than LEDs.

For ion imaging using commercial organic indicators the illumination intensity obtainable with LEDs is sufficient for all practical applications. In contrast, membrane potential imaging requires highintensity monochromatic light to resolve $\Delta \mathrm{F} / \mathrm{F}$ signals ranging between 0.1 and $10 \%$, which are sampled at sub-millisecond intervals. For this type of applications, LED illumination is inferior compared to the high monochromatic power obtainable with a laser. Currently, the light power obtained at the back-aperture of the objective with an LED is $<100 \mathrm{~mW}$ and is not truly monochromatic. Monochromatic illumination power of 200-300 mW can, however, be obtained using economic solid state lasers.

\section{Lasers}

Unlike in confocal, 2P, and TIRF applications the laser beam in voltage dye measurements is typically expanded and focussed onto the back-aperture of the objective to produce widefield illumination. In our experience uniform illumination cannot be efficiently obtained by simply using the epifluorescence port of a commercial microscope. Thus, whole-field laser illumination at $532 \mathrm{~nm}$, ideal for voltage imaging using the voltage sensitive dye JPW1114, was obtained using a dedicated mirror system from TILL Photonics inserted into the field-stop slider of an Olympus BX51 microscope (Holthoff et al., 2010; Canepari et al., 2010). In order to permit simultaneous secondary illumination from the epifluorescence port of the microscope, necessary to combine voltage imaging with other optical techniques, Cairn Research developed a dedicated system incorporating a beam expander and weak diffuser allowing direct epi-illumination from above the objective (described in Vogt et al., 2011a; 2011b). 
Laser illumination is typically noisier than LED illumination. Nevertheless, in the kilohertz range, economic solid-state lasers with fluctuations $<0.1 \%$ are now available, for instance the MLL series from CNI (Changchun, China) or the Excelsior from Newport-Spectra-Physics (Mountain View, CA). Other commercial providers for this type of economical laser are Laserglow (Toronto, Canada) and Cobolt (Stockholm, Sweden). As shown in Fig.1, voltage imaging using dyes that respond to changes in membrane potential with a shift in the absorption spectrum, such as the commercial styryl dyes, benefit greatly from using monochromatic laser light at the edge of the absorption spectrum. This dramatically increases the sensitivity of the measurement (Kuhn et al., 2004). Thus, $532 \mathrm{~nm}$ (Canepari et al., 2010) and $543 \mathrm{~nm}$ (Vogt et al., 2011a) lasers are ideal for voltage imaging experiments using JPW1114 or di-8-ANEPPS (Invitrogen, Carlsbad, CA).

A disadvantage of current affordable high power lasers is that they are based on diode pumped solid state (DPSS) technology and are consequently not sufficiently stable when triggered by a TTL pulse. Thus, useful laser illumination requires a mechanical shutter. In addition, although lasers are available at different wavelengths, the cost, power and stability are highly variable.

\section{Cameras}

Slow CCD cameras and fast photodiode arrays.

By the early nineties CCD camera technology had evolved to the point that calcium imaging measurements at acquisition rates in the order of tens of $\mathrm{Hz}$ were possible (see for example LasserRoss et al., 1991). These early CCD cameras could optimally operate with moderate light levels corresponding to hundreds to tens of thousands of photons per pixel. For voltage imaging, requiring acquisitions in the $\mathrm{kHz}$ range, photodiode arrays were preferred. Photodiode arrays have higher readout noise than CCDs but also have much higher well-depth and can operate at high levels of light corresponding to millions of photons per pixels per frame. These light levels render the read-out noise negligible, and instead allow small changes in signal to be monitored as the intrinsic photon noise is minimised. For this reason, they have been the optimal device for voltage imaging in extracellularly stained preparations and transmitted light measurements, such as invertebrate ganglia (see for example Zecevic et al., 1989) and brain slices (see for example Grinvald et al., 1982), where fractional changes of fluorescence are small but resting light level is high. They were also used for the first single cell studies (Zecevic, 1996) before being replaced by fast CCD cameras.

\section{Fast CCD and EMCCD cameras}


In the second half of the nineties, CCD cameras capable of acquisition rates of hundreds of $\mathrm{Hz}$ or faster became available (see for example Canepari \& Mammano, 1998; Mammano et al., 1999). Fast $(>100 \mathrm{~Hz})$ cameras with vastly differing resolution and well-depth are now commercially available. For example, RedshirtImaging offers the NeuroCCD SM256, a camera with 256X256 pixels of 600k $\mathrm{e}^{-}$ well-depth capable of acquiring up to 100 full frame/s, and the NeuroCCD SMQ, a camera with 80X80 pixels of $215 \mathrm{k} \mathrm{e}$ - well-depth capable of acquiring up to 2000 full frames/s. Because of its remarkable speed (up to $20 \mathrm{kHz}$ for binned sub-regions), the NeuroCCD SMQ was routinely used in single cell membrane potential recordings (Djurisic et al., 2004; Holthoff et al., 2010; Canepari et al., 2010; Foust et al., 2010) and in combined voltage and $\mathrm{Ca}^{2+}$ imaging recordings (Canepari et al., 2007; 2008; Milojkovic et al., 2007; Canepari \& Vogt, 2008). Other fast CCD cameras are available from Photometrics (Tucson, AZ), Princeton Instruments (Trenton, NJ), PCO (Kelheim, Germany), Hamamatsu Photonics (Hamamatsu City, Japan) and Teledyne DALSA (Thousand Oaks, CA).

Another advantage of modern CCD cameras is the high QE in the 500-700 nm range, $>60 \%$ for conventional sensors and $>90 \%$ for back-illuminated sensors, where the photons have an unrestricted path to the photo-active silica. Fast CCD cameras have read-out noise of a few up to a few tens of $\mathrm{e}^{-}$ and are optimal for applications with moderate light levels, typically in single cell voltage and ion imaging. This read-out noise, however, might be significant in applications with large signals but low levels of background fluorescence. This might be the case of long recordings, where exposure to highintensity illumination is limited by dye photo-bleaching, or when dyes with large dynamic range but low resting level are used (for instance the Fluo calcium indicators). In the past, the problem of amplifying the light signal without amplifying the read-out noise was solved by using intensified CCD cameras. Currently, the most practical solution is to use an electron multiplied (EM) camera. In an EMCCD camera, photoelectrons generated in each pixel are passed through a gain register to multiply them through a process of impact ionisation prior to digitisation. In this way, the signal reaching the digitiser is sufficiently large to render the effective read-out noise $<1 \mathrm{e}^{-}$. EMCCD cameras were first introduced by Andor Technology (Belfast, UK) who currently offers several back-illuminated EMCCD cameras with different numbers of pixels and well-depths running at different speeds. Many of the other companies offering CCD cameras (see above) also supply EMCCD cameras as an alternative for low-light or high speed applications. Currently, EMCCD cameras are the best option for ion imaging at low light levels (Coates et al., 2004) whereas for high levels of light, where the photon noise is dominant over the read-out noise then EM gain should not be used as it will offer no benefit and is in fact counterproductive as the gain register introduces statistical noise equivalent to square root of two. Many EM cameras incorporate a conventional read-out amplifier in addition to the EM amplifier so that the same camera is suitable for both high and low light measurements. 


\section{CMOS cameras}

For high light levels and high speed applications, PDAs were, until recently, the most commonly used systems. In these devices, each photodiode has a separate amplification system which limits the number of pixels and therefore the spatial resolution. For example, the NeuroPDA commercialised by RedshirtImaging has 464 pixels (diodes). More recently, the same performance (high well-depth $>10^{7}$ $\mathrm{e}^{-}$and $\mathrm{kHz}$ frame rate), but with a larger number of pixels $(>10,000)$ can be obtained using CMOS cameras. Fast CMOS cameras for high-light, high-speed imaging are available from RedshirtImaging and SciMedia (Costa Mesa, CA, the MiCAM02-CMOS system). High-speed and well-depth CMOS cameras are currently the best detection system to record optical membrane potential changes in the heart (Glukhov et al., 2010; Lang et al., 2011).

CMOS cameras are also available with lower well-depth and lower read-out noise, making this technology potentially superior for measurements at low or medium light intensity. This new generation of CMOS sensors with low read-out noise are usually referred to as "scientific" CMOS or sCMOS. Among those recently commercialised, the highest performing cameras are from Hamamatsu (model Orca Flash 4.0), PCO (Kelheim, Germany, model PCO.Edge), Andor (models Neo and Zyla). The sensors of these cameras have over $4 \mathrm{M}$ pixels with well-depth of 30k electrons and a read-out noise $<2$ electrons. The cameras mentioned above are all capable of acquiring 100 frames/s at full resolution but acquisition rates can be increased up to $25 \mathrm{kHz}$ for linear regions of interest (ROIs) in the centre of the sensor. Because high acquisition speeds can be obtained with a large number of pixels, the "effective" well-depth can be increased by binning the frames after acquisition making them suitable for applications where large pixels with high well-depth would traditionally be needed. As an interesting alternative for high speed recording RedShirt Imaging demonstrated a new CMOS camera at the 2013 Society for Neurosciences meeting. This camera has 128x128 pixels, 10,000 frames per second and a read noise of $<4 \mathrm{e}^{-}$. However, for extremely low light applications such as single molecule analysis, EMCCD cameras still have the upper hand. A comparison by Photometrics shows that up to about 10 photons per pixel, the $\mathrm{S} / \mathrm{N}$ is superior for EMCCD cameras.

\section{Discussion and future directions}

We have analysed here the illumination and detection systems that are currently available for invitro membrane potential and ion imaging. For illumination systems, we focussed on LEDs and solid state lasers as they represent the most practical solutions in nearly all applications. Lasers are more powerful and are always preferable where monochromatic light is needed. However, commercially available lasers may considerably vary in price and power according to the different wavelengths and 10 
cheap lasers cannot be reliably triggered, and thus require shutters to control illumination. This may change as diode lasers become available at more wavelengths and with higher powers. LEDs are usually preferable when illumination intensities up to $50 \mathrm{~mW}$ and spectral bands of $20 \mathrm{~nm}$ are sufficient. LEDs are cheap and can be TTL triggered making them ideal for applications where short exposure times are required. The technology of non-laser light sources is likely to advance in the next few years. It is worth mentioning here the recently available solid-state multi-colour light sources such as the Heliophor, commercialised by 89 North (Burlington, VT), and a range of illuminators from Lumencor (Beaverton, OR). These powerful (hundreds of $\mathrm{mW}$ ) light sources, that can be triggered by a TTL pulse, can be efficiently coupled to microscopes and offer an alternative to both LEDs, whilst still not providing as much power at the sample as lasers. We have summarised these different light sources in Table 1, showing the main characteristics as reported by manufacturers or as measured by us.

For light detection, an ideal device will always provide measurements with the best possible $\mathrm{S} / \mathrm{N}$, i.e. at the limit of photon noise. Currently, CCD, EMCCD and CMOS cameras have high QE and behave in an ideal manner for different ranges of light detection, i.e. they are ideal for different ion or voltage imaging applications. The number of pixels and the acquisition rates are correlated parameters that need to be taken into account. Whereas a lower number of pixels will always result in a compromise between spatial resolution (area of the preparation covered by a pixel) and total imaged area of the preparation, this option will typically provide a higher speed. In this respect, Fig. 3 illustrates the field of view, obtained with a $10 \mathrm{X}$ and $60 \mathrm{X}$ objective and no magnification, for some of the CCD, EMCCD, CMOS and sCMOS cameras currently available in the market. Table 2 summarises the most important characteristics of the same camera models. One point to note is that EM cameras from Photometrics and Andor have recently been released with significantly higher frame rates (Delta and Ultra respectively in the table), although this still lags behind the rates offered by sCMOS or dedicated high-speed CCDs.

A final issue that must be discussed is the potential progress beyond the "standard" imaging approaches based on uniform wide-field illumination and detection systems, and limited in quality by out of focus fluorescence and a lack of depth discrimination. Confocal and 2P microscopy have been extensively used to resolve signals from structures $<1 \mu \mathrm{m}$ such as dendritic spines. These measurements, however, required scanning the preparation with the laser and, in the case of confocal microscopy, with a pinhole. Scanning microscopy has the disadvantage that light exposure for each point is inversely proportional to the number of points at a given "frame" rate and, in addition, the QE of photomultipliers used in scanning microscopes is poor. Thus, confocal and $2 \mathrm{P}$ measurements are performed with a relatively low number of photons and consequently with a large photon noise. Both 
confocal and 2P microscopy are possible using CCD or CMOS cameras without scanning. For confocal microscopy, this can be obtained using a spinning disk. While the Yokogawa system (Yokogawa, Tokyo, Japan; also commercialised by Solamere Technology, Salt lake City, Utah) is currently the most used system, fast spinning disks are now available from Crest Instruments (Rome, Italy). For $2 \mathrm{P}$ microscopy, single spot illumination can be potentially replaced by holographic illumination allowing fluorescence excitation of well-defined patterns (Papagiakoumou et al., 2010), in this way permitting the use of a camera for recording fluorescence from the whole illuminated pattern. It is expected that careful optimisation of these two approaches will advance the state-of-the-art of both ion concentration and membrane potential measurements. 


\section{Glossary}

Charge coupled device (CCD)

Electrons ( $\mathrm{e}^{-}$)

Electron multiplying CCD (EMCCD)

Fractional change of fluorescence $(\Delta \mathrm{F} / \mathrm{F})$

Light emitting diode (LED)

Metal oxide semiconductor (CMOS)

Photodiodes array (PDA)

Quantum efficiency (QE)

Scientific CMOS (sCMOS)

Signal-to-noise ratio $(\mathrm{S} / \mathrm{N})$

Two-photon (2P)

\section{Acknowledgements}

We thank Antoine Delon for useful discussions on EMCCD and sCMOS cameras.This work was supported by the Agence Nationale de la Recherche (Grant Voltimagmicro, program Emergence-10 and Labex Ion Channels Science and Therapeutics). 


\section{References}

Augustine, G.J. (1994) Combining patch-clamp and optical methods in brain slices. J. Neurosci. Methods 54, 163-169.

Brown, J.E., Cohen, L.B., De Weer, P., Pinto, L.H., Ross, W.N. \& Salzberg, B.M. (1975) Rapid changes in intracellular free calcium concentration. Detection by metallochromic indicator dyes in squid giant axon. Biophys. J. 15, 1155-1160.

Canepari., M., Djurisic, M. \& Zecevic, D. (2007) Dendritic signals from rat hippocampal CA1 pyramidal neurons during coincident pre- and post-synaptic activity: a combined voltage- and calcium-imaging study. J. Physiol. 580, 463-484.

Canepari, M. \& Mammano, F. (1999) Imaging neuronal calcium fluorescence at high spatio-temporal resolution. J. Neurosci. Meth. 87, 1-11.

Canepari, M. \& Vogt, K.E. (2008) Dendritic Spike Saturation of Endogenous Calcium Buffer and Induction of Postsynaptic Cerebellar LTP. PLoS ONE 3, e4011.

Canepari, M., Vogt, K. \& Zecevic, D. (2008) Combining voltage and calcium imaging from neuronal dendrites. Cell. Mol. Neurobiol. 58, 1079-1093.

Canepari, M., Willadt, S., Zecevic, D. \& Vogt, K.E. (2010) Imaging Inhibitory Synaptic Potentials Using Voltage Sensitive Dyes. Biophys. J. 98, 2032-2040.

Coates, C.G., Denvir, D.J., McHale, N.G., Thornbury, K.D. \& Hollywood, M.A. (2004) Optimizing low-light microscopy with back-illuminated electron multiplying charge-coupled device: enhanced sensitivity, speed, and resolution. J. Biomed. Opt. 9, 1244-1252.

Djurisic, M., Antic, S., Chen, W.R. \& Zecevic, D. (2004) Voltage imaging from dendrites of mitral cells: EPSP attenuation and spike trigger zones. J Neurosci 24, 6703-6714.

Efimov, I.R., Nikolski, V.P. \& Salama, G. (2005) Optical imaging of the heart. Circ. Res. 95, 21-33.

Entcheva, E., Kostov, Y., Tchernev, E. \& Tung, L. (2004) Fluorescence imaging of electrical activity in cardiac cells using an all-solid-state system. IEEE Trans. Biomed. Eng. 51, 333-341.

Foust, A., Popovic, M., Zecevic, D. \& McCormick, D.A. (2010) Action potentials initiate in the axon initial segment and propagate through axon collaterals reliably in cerebellar Purkinje neurons. $J$. Neurosci. 30, 6891-6902.

Glukhov, A.V., Fedorov, V.V., Anderson, M.E., Mohler, P.J. \& Efimov, I.R. (2010) Functional anatomy of the murine sinus node: high-resolution optical mapping of ankyrin-B heterozygous mice. Am. J. Physiol. Heart Circ. Physiol. 299, H482-491.

Grinvald, A., Manker, A. \& Segal, M. (1982) Visualization of the spread of electrical activity in rat hippocampal slices by voltage-sensitive optical probes. J. Physiol. 333, 269-291. 
Harkins, A.B., Kurebayashi, N.\& Baylor, S.M. (1993) Resting myoplasmic free calcium in frog skeletal muscle fibers estimated with fluo-3. Biophys. J. 65, 865-881.

Helmchen, F. \& Konnerth, A. (2010) Imaging in Neuroscience: A Laboratory Manual. Cold Spring Harbour Laboratory Press, Cold Spring Harbour.

Holthoff, K., Zecevic, D. \& Konnerth, A. (2010) Rapid time course of action potentials in spines and remote dendrites of mouse visual cortex neurons. J. Physiol. 588, 1085-1096.

Homma R., Baker, B.J., Jin, L., Garaschuk, O., Konnerth, A., Cohen, L.B., Bleau, C.X., Canepari, M., Djurisic, M. \& Zecevic, D. (2009) Wide-field and two-photon imaging of brain activity with voltage- and calcium-sensitive dyes. Methods Mol. Biol. 489, 43-79.

Jin, L., Han, Z., Platisa, J., Wooltorton, J.R., Cohen, L.B. \& Pieribone, V.A. (2012) Single action potentials and subthreshold electrical events imaged in neurons with a fluorescent protein voltage probe. Neuron 75, 779-785.

Kuhn, B., Fromherz, P. \& Denk, W. (2004) High sensitivity of Stark-shift voltage-sensing dyes by one- or two-photon excitation near the red spectral edge. Biophys. J. 87, 631-639.

Lang D., Sulkin, M., Lou, Q. \& Efimov I.R. (2011) Optical mapping of action potentials and calcium transients in the mouse heart. J. Vis. Exp. 55, pii: 3275. doi: 10.3791/3275.

Lasser-Ross, N.,Miyakawa, H., Lev-Ram, V., Young, S.R. \& Ross W.N. (1991) High time resolution fluorescence imaging with a CCD camera. J. Neurosci. Meth. 36, 253-261.

Mammano, F., Canepari, M., Capello, G., Ijaduola, R.B., Cunei, A., Ying, L., Fratnik, F. \& Colavita, A. (1999) An optical recording system based on a fast CCD sensor for biological imaging. Cell Calcium 25, 115-123.

Milojkovic, B.A., Zhou, W.L., \& Antic, S.D. (2007) Voltage and calcium transients in basal dendrites of the rat prefrontal cortex. J. Physiol. 585, 447-468.

Miyawaki, A., Llopis, J., Heim, R., McCaffery, J.M., Adams, J.A., Ikura, M. \& Tsien RY. (1997) Fluorescent indicators for $\mathrm{Ca} 2+$ based on green fluorescent proteins and calmodulin. Nature 388, 882-887.

Mutoh, H., Perron, A., Akemann, W., Iwamoto, Y. \& Knöpfel, T. (2011) Optogenetic monitoring of membrane potentials. Exp. Physiol. 96, 13-18.

Papagiakoumou, E., Anselmi, F., Bègue, A., de Sars V., Glückstad, J., Isacoff, E.Y. \& Emiliani V. (2010) Scanless two-photon excitation of channelrhodopsin-2. Nat Methods 7, 848-854.

Ross, W.N., Salzberg, B.M., Cohen, L.B. \& Davila, H.V. (1974) A large change in dye absorption during the action potential. Biophys. J. 14, 983-986. 
Thomas D., Tovey S.C., Collins, T.J., Bootman, M.D., Berridge, M.J. \& Lipp P. A. (2000) Comparison of fluorescent $\mathrm{Ca}^{2+}$ indicator properties and their use in measuring elementary and global $\mathrm{Ca} 2+$ signals. Cell Calcium 28, 213-223.

Thomson, L.A. \& Hageage, G.J. (1975) Evaluation of excitation light sources for incident immunofluorescence microscopy. Appl. Microbiol. 30, 616-624.

Vogt, K.E., Gerharz, S., Graham, J. \& Canepari, M. (2011a) High-resolution simultaneous voltage and Ca2+ imaging. J. Physiol. 589, 489-494.

Vogt, K.E., Gerharz, S., Graham, J. \& Canepari, M. (2011b) Combining membrane potential imaging with L-glutamate or GABA photorelease. PLoS ONE 6, e24911.

Walton, R.D., Benoist, D., Hyatt, C.J., Gilbert, S.H., White, E., Bernus, O. (2010) Dual excitation wavelength epifluorescence imaging of transmural electrophysiological properties in intact hearts. Heart Rhythm. 7, 1843-1849.

Zecević, D. (1996) Multiple spike-initiation zones in single neurons revealed by voltage-sensitive dyes. Nature 381, 322-325.

Zecević, D., Wu, J.Y., Cohen, L.B., London, J.A., Höpp, H.P. \& Falk CX (1989) Hundreds of neurons in the Aplysia abdominal ganglion are active during the gill-withdrawal reflex. J. Neurosci. 9, 36813689. 


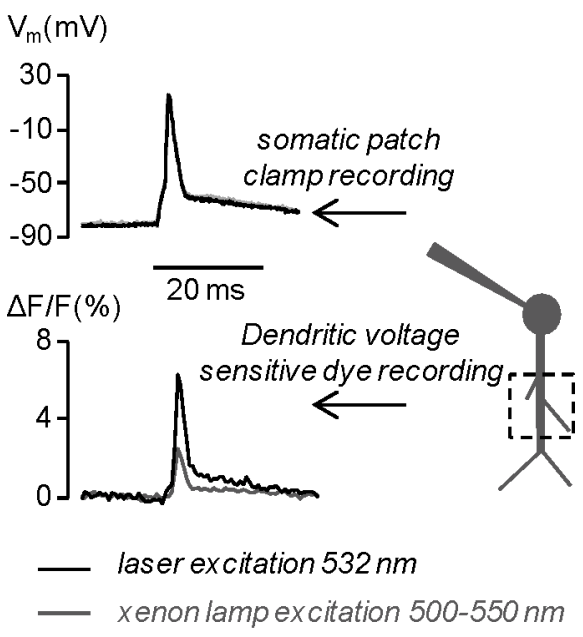

Fig.1. Comparison between $\Delta \mathrm{F} / \mathrm{F}$ signals associated with an action potential (top traces) from the dendrite of a CA1 hippocampal pyramidal neuron in slice; bottom gray trace: $\Delta \mathrm{F} / \mathrm{F}$ obtained by fluorescence excitation with the light of a xenon lamp filtered at $525 \pm 25$ $\mathrm{nm}$; bottom black trace: $\Delta \mathrm{F} / \mathrm{F}$ obtained by fluorescence excitation with a $532 \mathrm{~nm}$ laser. Modified from Canepari et al., 2010.
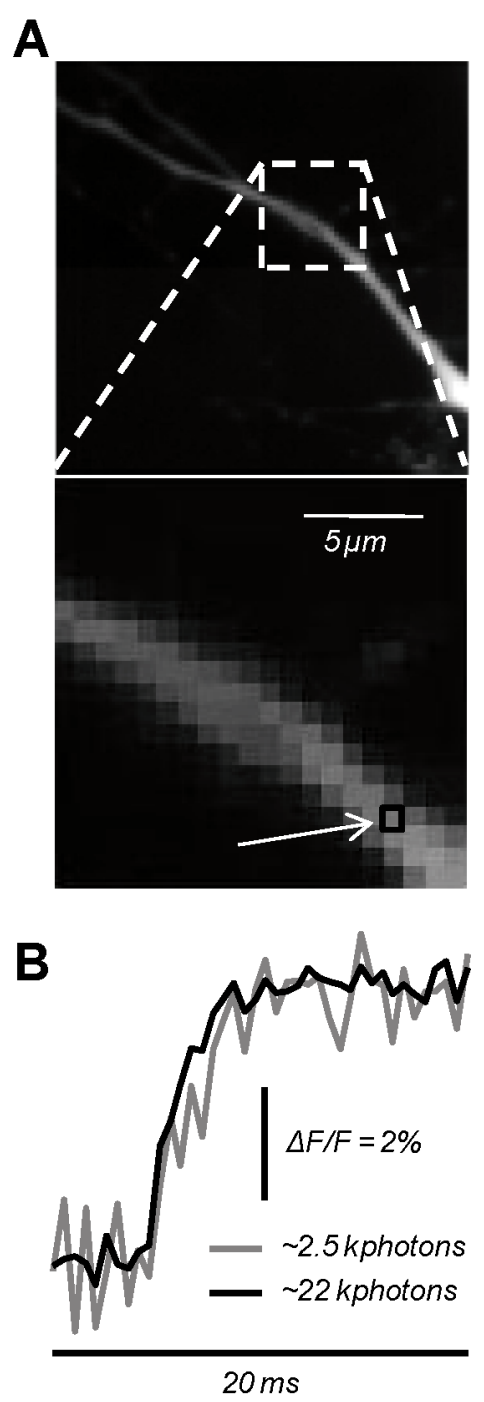

Fig.2. Photon noise limited $\mathrm{Ca}^{2+}$ recordings. (A) Dendrite of a neuron from a dissociated hippocampal culture filled with $200 \mu \mathrm{M}$ of the $\mathrm{Ca}^{2+}$ indicator Oregon Green BAPTA-1; top: full-frame taken with a NeuroCCD SMQ camera; bottom: zoom from the image above indicating a sample pixel. (B) $\Delta \mathrm{F} / \mathrm{F}$ from the pixel in A associated with an action potential; gray trace: single trial with 2,500 photons captured from the sample pixel; black trace: average of 9 trials (equivalent of $\sim 22,000$ photons from the sample pixel). In the single trial, read-out noise $\left(12 \mathrm{e}^{-}\right) \ll$ photon noise $\left(50 \mathrm{e}^{-}\right)$. 

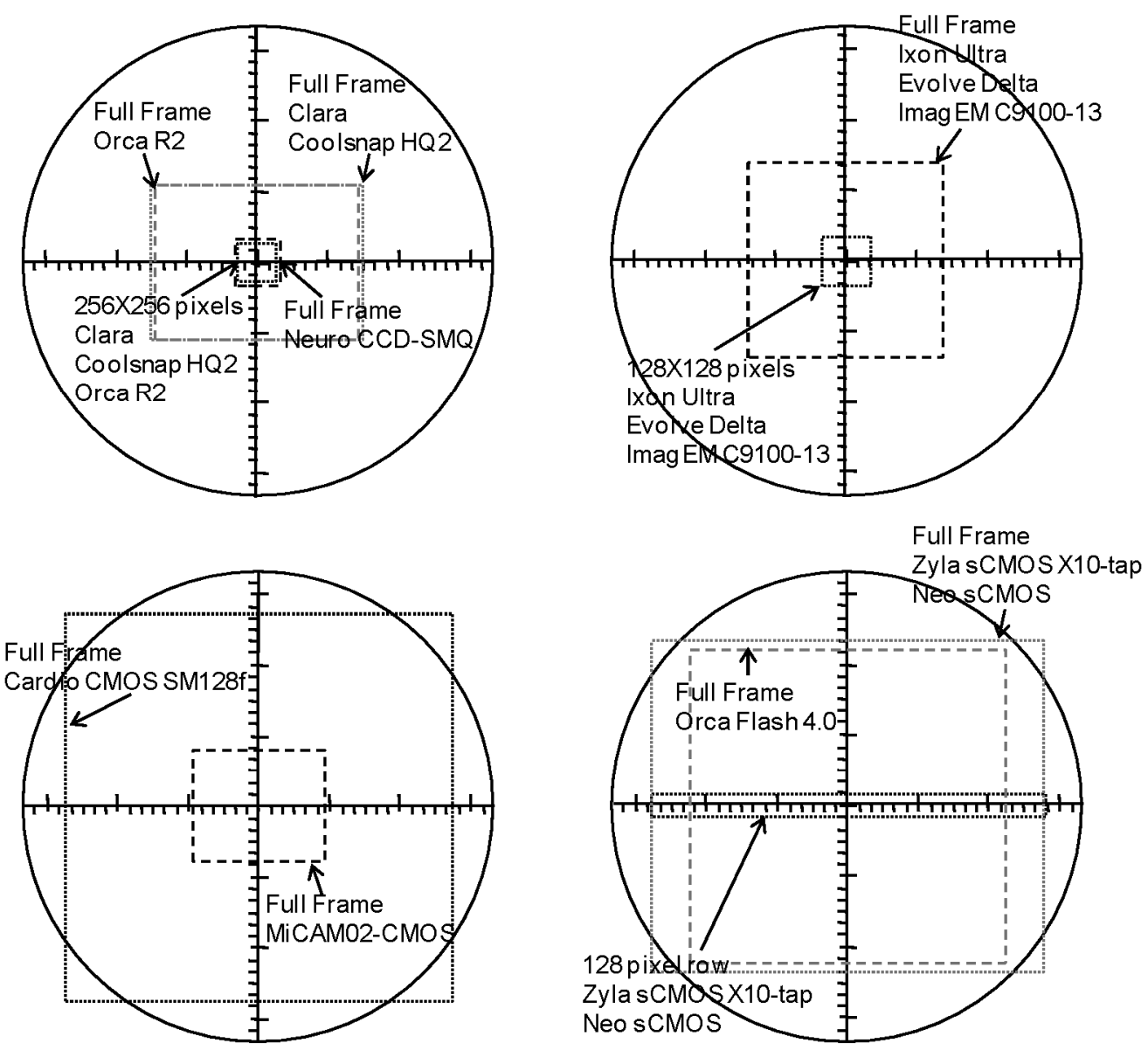

$-100 \mu \mathrm{m}$ (10X magnification)

$100 \mu \mathrm{m}$ (60X magnification)

Fig. 3. Field of view between the cameras listed in Table 2. This includes the regional field of view for CCD, EMCCD, CMOS and sCMOS cameras. 


\begin{tabular}{|c|c|c|c|c|c|}
\hline Type & Companies & Power $(\mathbf{m W})$ & Power @ obj (mW) & Wavelength width (nm) & Trigger \\
\hline LED & $\begin{array}{c}\text { Cairn, Thorlabs, Rapp } \\
\text { OptoElectronic }\end{array}$ & $100-900$ & $20-80$ & $20-200$ & Yes \\
\hline SS Laser & $\begin{array}{c}\text { CNI, Newport, } \\
\text { Laserglow, Cobolt }\end{array}$ & $100-500$ & $80-300$ & $1-2$ & No \\
\hline Solid state & North89, Lumencor & $150-750$ & $?$ & $20-40$ & Yes \\
\hline
\end{tabular}

Table 1. The up-to-date principal light sources for voltage and ion imaging with some major manufacturing companies, light power as provided by manufactures, power at back-aperture of the objective measured by us, typical wavelength width of the spectrum and whether or not can be reliably triggered by a TTL pulse in less than $1 \mathrm{~ms}$. Each light source has fluctuations at $10-10 \mathrm{k} \mathrm{Hz}<0.1$ as measured by us.

\begin{tabular}{|c|c|c|c|c|c|}
\hline CCD & No. Pixels & $\begin{array}{l}\text { Pixel size } \\
\quad(\mu \mathrm{m})\end{array}$ & WD (e-) & RON (e-) & Speed (frames/s) \\
\hline $\begin{array}{l}\text { CoolSnap HQ2 } \\
\text { (Photom etrics) }\end{array}$ & $1392 \times 1040$ & $6.45 \times 6.45$ & 16000 & 5.5 & Full frame $-11,512 \times 512$ w ith $4 \times$ binning -57 \\
\hline Clara (Andor) & $1392 \times 1040$ & $6.45 \times 6.45$ & 18000 & 6.5 & Full frame $-11,512 \times 512 w$ ith $4 \times$ binning -52 \\
\hline Orca R2 (Ham am atsu) & $1344 \times 1024$ & $6.45 \times 6.45$ & 18000 & 6 & Full frame $-16,512 \times 512$ w ith $4 x$ binning -65 \\
\hline $\begin{array}{l}\text { NeuroCCD SMQ } \\
\text { (Redshirt Im aging) }\end{array}$ & $80 \times 80$ & $24 \times 24$ & 215000 & 12 & 2000 \\
\hline \multicolumn{6}{|l|}{ EMCCD } \\
\hline Ixon Ultra (Andor) & $512 \times 512$ & $16 \times 16$ & 160000 & $<1$ w ith EM gain & Full frame $-56,128 \times 128$ pixels w ith CSM -595 \\
\hline $\begin{array}{l}\text { Evolve Delta } \\
\text { (Photom etrics) }\end{array}$ & $512 \times 512$ & $16 \times 16$ & 180000 & $<1$ w ith EM gain & Full frame-62.5, 235@128x128 pixels \\
\hline $\begin{array}{l}\text { Im agEM C9100-13 } \\
\text { (Hamamatsu) }\end{array}$ & $512 \times 512$ & $16 \times 16$ & 370000 & $<1 \mathrm{w}$ ith EM gain & Full frame-31.9,105@128 pixel row s \\
\hline \multicolumn{6}{|l|}{ CMOS } \\
\hline $\begin{array}{l}\text { Cardio CMOS SM128f } \\
\text { (Redshirt Im aging) }\end{array}$ & $128 \times 128$ & $128 \times 128$ & 100000000 & 400 & Full frame - 10000 \\
\hline $\begin{array}{l}\text { MiCAM 02-CMOS (Sci } \\
\text { Media) }\end{array}$ & $188 \times 160$ & $30 \times 30$ & 450000 & $<150$ & Full frame -1700 \\
\hline \multicolumn{6}{|l|}{ Scientific CMOS } \\
\hline $\begin{array}{l}\text { Zyla sCMOS X10-tap } \\
\text { (Andor) }\end{array}$ & $2560 \times 2160$ & $6.5 \times 6.5$ & 30000 & 1.2 & Full frame - 100, 1639@128x128 pixels \\
\hline Neo sCMOS (Andor) & $2560 \times 2160$ & $6.5 \times 6.5$ & 30000 & 1 & Full frame-100, 1616@128x128 pixels \\
\hline $\begin{array}{l}\text { Orca Flash } 4.0 \\
\text { (Ham amatsu) }\end{array}$ & $2048 \times 2048$ & $6.5 \times 6.5$ & 30000 & 1.2 & Full frame-100, 1603@ 128x128 pixels \\
\hline
\end{tabular}

Table 2. List of selected cameras for voltage or ion imaging; all cameras except the MiCAM02-CMOS have high QE (>60\%) at 500-700 nm and a dynamic range >4000. Reported characteristics were given by manufacturers. 